\title{
M87 Solves the Problem of AGN
}

\author{
S. G. Iskudarian
}

Byurakan Astrophysical Observatory, Byurakan, 378433, Armenia

\begin{abstract}
The example of M87 shows that AGN are the most powerful units of protogalactic matter, which has built the large scale Universe during parts of the first second of the Big Bang. For fulfilment of such a mission it had to possess properties of elementary particles.
\end{abstract}

The active nucleus of M87 is the active nucleus of Our Supergalaxy (Iskudarian 1993). M87 itself is that "globular cluster" in the center of the Supergalaxy, a similar image of which is observed in the centers of Our Galaxy and the Andromeda nebulae in small scales (Waldrop 1985, Iskudarian 1995). M87 is the nearest cD galaxy (Suchkov 1988), it is the nearest "void" (Iskudarian 1995) with its immediate environment. Recent investigations showed that voids are not voids at all (Peterson et al. 1991). Their blue centers are surrounded by faint elliptical galaxies. In the case of M87 globular clusters rich population is in the role of superdwarf ellipticals, which does not contradict the reality.

M87 gave rise to a closed looplike superstring in Our Supergalaxy (Iskudarian) in the form of the rich group of galaxies N94 (Huchra \& Geller 1983), which is connected with the other rich group of galaxies N106 physically and, it seems, was ejected from the entrails of the last one (in a protogalactic state certainly). Looking on Fig. 1a, b of Iskudarian (1993) one can see the connection of those two groups very well. Such a picture could form only during parts of the first second of the Big Bang, with the high energies released at the high temperatures. In such conditions takes place rather unusual phenomena in the micro world. For example, the sudden birth of a new particle or the decay of a nonstable particle in a heavy shower of other particles. There can meet particles also which combine in themselves characters of two quite different particles, so-called "mad" unity (Iskudarian 1997, Davies 1985). Observations show the existence of analogous processes in the macro world - in the world of galaxies. One may think with great conviction that those properties of elementary particles were kept in protogalactic matter as its "genes" (Iskudarian 1997). And AGN are those agents which carried the huge energies of the Big Bang to the remote corners of the observed Universe, drawing with its treks the large scale structure of the Universe. Later on, being centers of supergalaxies, and so the other potential centers of activity, they have built and build at present new structures in the form of new generations of stellar populations. The composite structure of the Virgo cluster is the best evidence of these last thoughts (Bingelli et al. 1987).

Undoubtedly, the thoughts expressed here need further study, investigation, and discussion. Nevertheless, it is clear that activity of AGN in its outward displays is diverse and many-coloured, but in the basis, there is the general 
regularity which acts in the micro and macro worlds of the Universe, ensuring by this way the unity of the Universe. It is the origin, formation, and ejection of the first type of stellar population from the entrails of the second one (Iskudarian $1993,1996)$.

\section{References}

Bingelli,B., Tammann, G.A., \& Sandage, A.R. 1987, AJ, 94, 251

Davies, P. 1985, Superforce, New York

Huchra, J.P., \& Geller, M.J. 1983, ApJS, 52, 61

Iskudarian, S.G. 1993,"Is M87 the active nucleus of Our Supergalaxy?", International workshop on "Galaxy Clusters and Large Scale Structures in the Universe" Sesto Pusteria (Bolzano, Italy)

Iskudarian, S.G. 1995, "Similar structures-similar formations of large and small scales", International workshop on "Observational Cosmology: from Galaxies to Galaxy Systems", Sesto Pusteria (Bolzano,Italy)

Iskudarian,S.G. 1995, "The nearest "void?", International workshop on "Observational Cosmology: from

Galaxies to Galaxy Systems", Sesto Pusteria (Bolzano,Italy)

Iskudarian, S.G. 1996, "The Unity of the Universe", International workshop on "Hubble deep field", Baltimore, USA

Iskudarian, S.G. 1997,"The Universe of micro and macro scales", Meeting Astro4, Moskow

Iskudarian,S.G. 1998, "An example of closed looplike superstring", (unpublished work)

Peterson, B.A., D'Odorico,S., Tarenghi M., \& Wampler, E.J. 1991, The Messenger, N64, 1

Suchkov, A.A. 1988, "Familiar and Mysterious Galaxies", "Science", Moskow

Waldrop, M.M. 1993, Science, 230, N4722, 158 